\title{
Underlying the mechanisms of changes in cancer prevalence among the U.S. Medicare beneficiaries: contributions of incidence, survival, and ascertainment at early stages.
}

Igor Akushevich ( $\square$ igor.akushevich@duke.edu )

Duke University https://orcid.org/0000-0003-3471-7846

Arseniy Yashkin

Duke University Social Science Research Institute

Mikhail Kovtun

Medanta Duke Research Institute

Anatoliy I. Yashin

Medanta Duke Research Institute

Julia Kravchenko

Duke University School of Medicine

\section{Research Article}

Keywords: Time trends, Decomposition, Partitioning, Incidence, Survival, Stage ascertainement

Posted Date: February 15th, 2022

DOI: https://doi.org/10.21203/rs.3.rs-1274934/v1

License: (c) (1) This work is licensed under a Creative Commons Attribution 4.0 International License.

Read Full License 


\title{
Underlying the mechanisms of changes in cancer prevalence among the U.S. Medicare beneficiaries: contributions of incidence, survival, and ascertainment at early stages.
}

\author{
I Akushevich', A.Yashkin ${ }^{1}$, M. Kovtun ${ }^{1}$, A. I. Yashin ${ }^{1}$, J Kravchenko ${ }^{2}$ \\ ${ }^{1}$ Center for Population Health and Aging, Duke University, Durham, NC, USA \\ ${ }^{2}$ Department of Surgery, Duke University School of Medicine, Duke University, Durham, NC, \\ USA
}

Corresponding author: Igor Akushevich, email: igor.akushevich@duke.edu

\begin{abstract}
:
Purpose: To quantitatively evaluate contributions of trends in incidence, relative survival, and stage at diagnosis to the dynamics in the prevalence of major cancers (lung, prostate, colon, breast, urinary bladder, ovaries, stomach, pancreas, esophagus, kidney, liver, and skin melanoma) among older U.S. adults age 65+.

Methods: Trend partitioning was applied to the Surveillance, Epidemiology, and End Results Program data for 1973-2016.

Results: Growth of cancer prevalence in older adults decelerated or even decreased over time for all studied cancers due to decreasing incidence and improving survival for most of cancers, with a smaller contribution of the stage at cancer diagnosis. Changes in prevalence of cancers of lung, colon, stomach, and breast were predominantly due to decreasing incidence, increasing survival and more frequent diagnoses at earlier stages. Changes in prevalence of some other cancers demonstrated adverse trends such as decreasing survival in localized and regional stages (urinary bladder and ovarian) and growing impact of late-stage diagnoses (esophageal cancer).

Conclusion: While decelerating or decreasing prevalence of many cancers were due to a beneficial combination of decreasing incidence and increasing survival, there are cancers for which decelerating prevalence is due to the lack of improvement in their stage-specific survival and/or increasing frequency of diagnosis at advanced stages. Overall, if the observed trends persist, it is likely that cancer burden due to its prevalence in older U.S. population will be lower than it was projected in earlier studies.
\end{abstract}

Keywords: Time trends, Decomposition, Partitioning, Incidence, Survival, Stage ascertainement 


\section{Introduction}

More than half of all cancer survivors in the United States are older than 65 years and the number of older survivors is rising [1] thus posing a challenge to the delivery of quality cancer care [2]. Changes in cancer prevalence result from the interplay of the dynamics of cancer incidence, survival, and stage ascertainment; therefore, evaluation of the mechanisms of changes in prevalence over time provides valuable information on the effectiveness of cancer prevention, early diagnosis, and treatment. Furthermore, prevalence is a measure of disease burden in the population that allows for the planning of health care needs [3]. Information on cancer burden in the older U.S. population can be used in analyses of health expenditures, morbidity, quality of life, and life expectancy. It is increasingly recognized that older survivors have complex healthcare needs, but these are poorly understood [4]. At present, little is known about the burden of cancer and comorbidity among the older adults (i.e., especially those aged 85+) and about the dynamics of this burden over time [5].

While studies on prevalence are common for chronic diseases with a long duration (e.g., diabetes [6], Alzheimer's disease [7], or osteoarthritis [8]), studies on cancer prevalence are less common with more emphasis placed on cancer incidence or mortality, especially in older U.S. adults. This could be partially due to the complexity of interpretating prevalence trends which result from three distinct interrelated processes: dynamics in cancer incidence, survival, and the average stage at time of diagnosis. The partitioning analysis [9-12] used in this study, generates models of cancer prevalence, incidence, survival and stage ascertainment and then provides quantitative estimates of the relative fractions of the contributions of the above components to the total change in cancer prevalence. Together, changes in the relative magnitudes of these components provide a quantitative picture describing the epidemiologic causes of prevailing prevalence trends and how these have changed over time in response to changes in screening, treatment and exposure to risk-related factors (e.g., smoking, asbestos exposure etc.). This allows researchers

to identify both positive trajectories in the epidemiology of a specific cancer (e.g., falling incidence, increasing survival, higher proportion of cases diagnosed a lower stages) as well as potential areas of concern requiring urgent attention from the medical and health policy communities. In a time when improved cancer survivorship is becoming a prominent challenge 
to the U.S. healthcare system, such estimates can provide information invaluable in preparing for the expected increase in cancer survivors [13, 14]. Our study focuses on the U.S. population of older adults age $65+$ because this population has the highest cancer prevalence among all age groups and, due to the high rates of chronic morbidities in this age group, represents the highest potential challenge for maintaining the health and wellbeing of cancer survivors.

\section{Data and Methods}

Data. The SEER data for 1973-2016 were used in this study. Data before 1992 were used as a look-back period to calibrate the model. SEER*Stat software was used to evaluate age-adjusted prevalence that was used for comparisons with prevalence proportions calculated using the partitioning approach. The following codes of SEER site records ICD-O-3 were used for analysis: cancer of lung and bronchus (C340-C349), breast (C500-509), prostate (C619), colon (C180, C182-C189, C199), pancreas (C250-C259), urinary bladder (C670-C679), esophagus (C150-C159), liver (C220), kidney (C649, C659), stomach (C160-C169), ovarian (C569), and skin melanoma (C440-C449).

\section{Partitioning Analysis}

The partitioning approach [9-12] is based on an explicit representation of disease prevalence with no simplifying assumptions. The full mathematical derivation of the partitioning approach and its application to lung cancer is available in the study by Akushevich et al. [11]. In brief, the method predicts cancer prevalence and decomposes (or partitions) their time trends into their constituent components by calculating the relative impact each component has on the overall trend as well as inter-temporal changes in the strength and direction of these impacts. Specific outcome measures are age-specific and age-adjusted prevalence; the constituent components are incidence, relative survival, and frequency of cancer stage at diagnoses.

The model of cancer prevalence is based on the idea that the probability of being prevalent at a given age requires being incident before and survival after that age. Time trends in age-adjusted prevalence are defined as their derivatives with respect to time $y$. Explicit calculation of time 
trends in age-adjusted prevalence results in $P^{\prime}(y) / P(y)=T_{i n c}(y)+T_{\pi}(y)+T_{S}(y)$, representing changes in incidence, cancer stage at the time of diagnosis, and relative survival, respectively. Three stages (localized regional and distant) are defined by SEER or modeled from AJCC stages (for prostate cancer). Explicit expressions for partitioning components of cancer age-adjusted prevalence are given in Equations 5 in the study by Akushevich et al. [11]. Each component is to be interpreted as the rate of change at any point in time (increasing if $>0$, and decreasing if $<0$ ) with the magnitude of the effect indicating the speed of the change. The sum of the contributions adds to $+100 \%$ if the decomposed prevalence is increasing and to $-100 \%$ if the decomposed prevalence is decreasing at a given point in time.

\section{Model Estimation}

The quantities of interest (i.e., $T_{i n c}(y), T_{\pi}(y)$, and $\left.T_{S}(y)\right)$ are expressed in terms of the derivatives of the respective functions with respect to time. In our approach, we used explicit analytic parameterizations for all functions for which derivatives are needed. Therefore, we calculated the derivatives analytically. This allowed us to avoid dealing with possible numeric instabilities occurring when derivatives are evaluated numerically. For any function, we used a two-stage approach: i) we parameterized the function and fitted data for each year of diagnosis and ii) we used B-splines to model relationships between year-specific model parameters and evaluate $y$-dependence of the function. B-splines allow explicit calculation of derivatives without requiring additional simplifying assumptions. The analysis involved the design and estimation of three models: i) for the incidence rate, ii) for frequencies of stage at diagnosis, and iii) for the probability of relative survival after cancer diagnosis. The distribution of age and time at cancer onset was modeled using the Armitage-Doll model with additional individual predisposition modeled by gamma or inverse Gaussian distributions (for incidence) [15], the Weibull model for time after disease onset (for relative survival), and the quadratic function of age dependence (for frequencies of stage at diagnosis). Time trends were modeled by estimating model parameters for each year and applying B-splines to fit the time patterns of obtained parameters. We note that the Weibull model for relative survival was parameterized in terms of $\mu$ and $\sigma$ exactly as described in refs. $[11,12]$ for all cancer sites except of cancers of prostate and breast, and skin melanoma 
for which we used the model in terms of $\alpha$ and $\sigma$. The latter model allows for relative survival for certain cancer sites exceeding $100 \%$ (or >1.00): i.e., when survival of patients with certain early-stage cancers was better than survival of individuals in general U.S. population aged 65+, that is observed for these cancer sites.

All three models (incidence, stage frequency, and relative survival) were estimated separately and obtained fits were used to construct the model for cancer prevalence for each cancer site. To assess the goodness-of-fit of our model, age-patterns of cancer incidence (averaged over 19922016) and time patterns of age-adjusted measures for incidence, stage frequencies, and relative survival are shown in the 12 cancer-specific panels of Supplementary Figure 1. The predicted prevalence model calculated based on partitioning approach was compared to the estimates provided by SEER (available through SEER*Stat software). The SEER*Stat estimates for cancer prevalence were generated using two alternative lookback periods: 18 years, the longest period available for all years of cancer initial diagnosis; and up to 23 years, the longest period available in the data, although not for all years of cancer initial diagnosis.

\section{Results}

The trends of cancer-specific prevalence were calculated using the partitioning approach and compared to corresponding estimates provided by the SEER*Stat tool (Figure 1). Adding the look-back years in SEER*Stat estimates increases the prevalence by identifying additional patients who were still surviving persons after being diagnosed with a cancer over the look-back period. Our model, which calculates cancer prevalence outside the bounds of the look-back periods, yielded the highest prevalence levels. The agreement between empirical data and our model was good for all cancers: the shape of the model reflected respective empirical patterns for 18 years, with differences in magnitude reflecting the effect of accumulated prevalence from earlier years as expected [12].

Cancer prevalence estimated from our model monotonically increased for all cancer sites with exceptions for four cancers for which a maximum in prevalence was reached with subsequent decline. These were: cancers of colon (with maximum in 1999), urinary bladder (2008), ovarian 
(2008), and prostate (2010) (Figure 1). Cancers of pancreas, esophagus, liver, kidney, and melanoma had the most pronounced increases in prevalence over time.

The relative rate of change (i.e., $\left.P^{\prime}(y) / P(y)\right)$ is shown in Figure 2 together with three components, incidence, relative survival, and frequencies of stage at diagnoses which, together, add up to the total. The magnitude of the increase in prevalence goes down for all cancers with two evident exceptions, pancreas and kidney cancer, for which there exist periods of acceleration in prevalence growth: 1992-2008 for kidney and 1992-2013 for pancreas cancer. Supportive information on explicit trends of partitioning components obtained empirically and in the model is shown in Supplementary Figure 1.

Empirical trends showed decreasing age-adjusted (65+) incidence rates for cancers of lung, colon, stomach, and ovarian over the entire study period. Only skin melanoma had increasing incidence rates during entire study period. Other cancers had periods of both increasing and decreasing incidence rates (Supplemental Figure 1). The results of partitioning analysis (Figure 2) show that changes in incidence rates were the main contributors to the deceleration of prevalence growth. Most substantially incidence contributed to prevalence deceleration for cancers of lung, colon, urinary bladder (since 2005), stomach, ovaries, and breast (since 2010). Decline in the prevalence for several cancers, colon, bladder (since 2005), ovarian (since 2008) and, prostate (since 2011) was the result of the effect of incidence trends. Although trends in incidence differ, the rate of change in the incidence effect goes down for most of the studied cancers (Figure 2), although for cancers of kidney, liver, and pancreas the decline starts on or about 2005.

Empirical trends of 1- and 3-year survival showed gradual improvements in relative survival among older adults age 65+ for all cancers except urinary bladder and ovarian (for local and regional stages), liver (for regional and distant stages), and prostate (local and distant) (Supplemental Table 1). Partitioning analysis showed that the relative survival from all cancers had a beneficial trend and pushed cancer prevalence up, though improvements in relative 
survival (Figure 2). However, the impacts of better survival on the prevalence trend were slowing down over time for all cancers except kidney cancer. Most rapid decline in the effect of survival was detected for urinary bladder and ovarian cancers.

Empirical trends showed that over time, in older adults age 65+, cancers of breast, prostate, skin melanoma, colon, urinary bladder, liver, and kidney were more often diagnosed at early (i.e., localized) stages (Supplemental Table 1). However, cancers of lung, pancreas, stomach, ovaries and esophagus were more often diagnosed at distant rather than in localized or regional stages, and this trend was growing for lung, stomach and especially esophageal cancers (Supplemental Table 1). Compared to the contributions of incidence and relative survival, the contribution of stage at ascertainment to the dynamics of cancer prevalence were less pronounced for most studied cancers. This contribution was minimal for cancers of colon, urinary bladder, prostate, and skin melanoma, but the effect of stage at ascertainment on dynamics of cancer prevalence overcame the impacts of incidence for pancreatic cancer (since 2013) and was comparable to impact of relative survival for kidney cancer (Figure 2). A disadvantageous and relatively strong in magnitude effect of stage ascertainment was detected for esophageal cancer (i.e., the impacts of cancer being diagnosed at advanced stage) (Figure 2).

\section{Discussion}

Using partitioning approach [11, 12], we quantitatively evaluated contributions of cancer incidence, relative survival, and early-stage diagnoses to the changes in cancer prevalence in older U.S. population aged 65+. In this study, partitioning of prevalence was analyzed together with empiric patterns of cancer incidence and stage-specific relative survival thus providing more detailed information on the mechanisms of changes in prevalence of various cancers in the U.S. For all studied cancers, growth of prevalence decelerated over time and for cancers of colon, urinary bladder, ovaries, and prostate, the prevalence passed its respective maximum resulting in declines over recent years. Trends in cancers of lung, colon, stomach, and breast reflect positive reasons for the observed prevalence change: decreasing incidence and increasing 
survival together with increases in diagnoses at earlier stages over time. However, prevalence trends of certain cancers reflect adverse and sometimes alarming patterns. Specifically, cancer of urinary bladder shows decreasing survival in localized and regional stages, ovarian cancer shows decreasing survival in localized and regional stages, although with an increase in earlystage diagnoses, and esophageal cancer shows an increasing impact of late-stage diagnoses.

Why it is important to understand underlying mechanisms of cancer trends in older adults. Aging of the U.S. population is a well-recognized factor contributing to cancer burden [16, 17]; therefore, timely information on cancer burden in older U.S. adults can be used for further optimization of cancer prevention, early diagnosis, and treatment strategies in this growing sector of the population. At present, information on cancer site-specific epidemiologic components (such as incidence, survival, and diagnosis at early vs. advanced stage) that define the dynamic of cancer prevalence is sparse. However, this information is important for understanding the mechanisms behind changes in cancer prevalence, evaluation of health expenditures and cancer burden among older U.S. adults. In addition to higher cancer prevalence, older patients often have functional impairment, multiple coexisting diseases, cognitive impairment, and a higher risk of treatment side effects that could lead to the choices toward less aggressive/effective treatment [18]. Despite their numbers, older adults continue to be understudied, a point emphasized by the Institute of Medicine in addressing the challenges in delivery of a quality cancer care [19]. Cancer patients age 65+ were largely excluded from clinical trials, precluding opportunities to develop a greater insight about the unique needs of older patients, including long-term survivors $[2,19,20]$. Therefore, it is crucial to investigate cancer outcomes and their recent trends in older patients to obtain an important information on this growing group of population that remains at increased risk of social, physical, and mental vulnerability.

Dynamics of cancer prevalence among older U.S. adults. The main driving forces underlying the prevalence trends observed in our study were a deceleration in incidence increase and improved relative survival for the majority of cancers studied. If current trends persist, we expect a substantial deceleration in the prevalence of many cancers in the U.S. population of older adults age $65+$ within the next decades. For many cancers dynamics of prevalence have slowed 
down (lung, stomach, and breast) or even started to decline (colon, prostate, ovarian, and urinary bladder). These trends are not in a full agreement with a recent projections suggesting that the prevalence of cancer survivors will increase from 15.5 million to 26.1 million (across all age groups) from 2016 to 2040, with the largest growth of cancer survivors in the older population [2]. The same study estimated that compared to 1975, the 2040 projected cancer prevalence will be 6-fold higher for those aged 65-74 years, 10-fold higher for ages 75-84 years, and 17-fold higher for ages 85+[2]. However, this study held the rates of cancer incidence and survival constant for the whole period of the projection, while in our approach we incorporated the dynamics of cancer incidence, relative survival, and stage at cancer diagnosis when analyzing changes of prevalence of each cancer.

Dynamics of cancer incidence and its role in cancer prevalence trends. Deceleration of growth of cancer prevalence obtained in our study can be explained, to a great extent, by the deceleration of the increase or actual decrease of cancer incidence over time such as observed for cancers of lung, colon, stomach, ovaries, urinary bladder, and breast. Effectiveness of ongoing preventive strategies substantially contributed to the deceleration of incidence observed over recent decade. They include, but not are limited to, decreasing smoking prevalence (affects risk of lung, urinary bladder, kidney, liver, colon, and stomach cancers, with the respective percent of smokingattributable deaths estimated at $80.2 \%, 44.8 \%, 16.8 \%, 23.6 \%, 9.7 \%$, and $19.6 \%$, respectively [21]), implementation of gastric cancer prevention programs that includes screening for Helicobacter pylori infection and its treatment [22], and decreasing prevalence of hepatitis B infection and its increasing vaccination rate (for liver cancer [23]). However, persisting or even increasing prevalence of metabolic syndrome, obesity, and hepatitis $\mathrm{C}$, as well as a poor diet, low physical activity, and other risk factors still slow the deceleration of cancer incidence.

Dynamics of relative survival and its role in cancer prevalence trends. The trends of relative survival obtained in our study were beneficial for all studied cancers, though improvement in relative survival has slowed down within recent decade for many cancers. The beneficial trends of relative survival could decline over time because of accumulation of cancer patients who benefited from increased survival in earlier years and, therefore, died later [12]. For example, it 
has been shown that the number of cancer survivals among older U.S. adults grew over time, with gender-specific differences widening at older ages: there was 5\%-8\% more male than female cancer survivors at ages 70-79, 11\% more at ages 80-89, and 12\% more at age 90+ (with $37 \%$ of men aged $90+$ being cancer survivors [2]. Slowing improvement or even decline in relative survival detected for several cancers (e.g., bladder, liver, ovarian) in our study can be the result of the accumulation of delayed mortality in individuals diagnosed early in the study timeperiod and benefiting from improved survival at the time of their diagnosis. Targeted partitioning of cancer mortality [12] would be required to further analyze this hypothesis.

The contributions of slowing improvements in survival to dynamics of prevalence were higher for cancers of prostate, ovaries, and urinary bladder. These effects were also observed for empiric trends of stage-specific survival of these cancers: e.g., decreasing trends of prostate cancer survival in epidemiologic analysis for localized and distant stages (Supplemental Figure 1). Another study showed decreases in survival (non-stage specific) among patients with prostate cancer from 2006/2010 to 2011/2016: a 5-year survival decreased for 1.3\% for ages $65-79$ and for $7.2 \%$ for patients aged $80+$ [24]. It has been suggested that lower survival at older ages might be due to a more rapid development of resistant prostate cancer, reduced ability to receive cancer therapies, and the impact of comorbidities [24, 25]. However, that cannot explain the changes in prostate cancer survival among older patients during the recent decade, as well as the negative dynamics of survival at localized stages observed in our study.

The trends of ovarian cancer survival among females age $65+$ show that while there are positive contributions of the decreasing role of incidence and the increasing frequency of earlier stage diagnoses of ovarian cancer, the relative survival of patients with localized and regional tumors actually decreased since 2003. While publications on survival trends among older patients with ovarian cancer remain sparse, a recent study showed that females age 75+ with ovarian cancer had stage-appropriate cancer surgery $(\mathrm{OR}=0.58$, 95\%CI-0.40-0.83) and got multi-agent chemotherapy $(\mathrm{OR}=0.27,95 \% \mathrm{CI}=0.17-0.41)$ [26] less often than patients younger than 50 years old. 
We also observed decreasing 3-year survival among older patients with localized or regional cancer of urinary bladder. This is in agreement with a study showing that treatment developments over the recent three decades did not result in improvements in mortality from localized or regional cancers of urinary bladder, with this negative trend being especially pronounced among older patients [27]. Better management algorithms and more effective treatment strategies are necessary to improve outcomes for such individuals.

Stage at cancer diagnosis and its role in the dynamics of cancer prevalence. Compared to contributions of relative survival and incidence, changes in stage at cancer diagnoses contributed less to the dynamics of cancer prevalence for most of cancers in our study except for cancers of pancreas; kidney and stomach (through increasing frequency of early-stage diagnoses); and esophagus (through increasing frequency of late-stage malignancies). Information on trends of stage at diagnosis for these cancers is sparse, especially for older patients. Cancers of pancreas and kidney are rarely diagnosed at early stages and screening for these cancers is not feasible for the general population [28, 29]. Screening for pancreatic cancer is recommended for high-risk groups starting from age 50 (with the use of endoscopic ultrasound and/or magnetic resonance imaging) [28, 30], and screening for kidney cancer (with the use of computer tomography or magnetic resonance imaging) is recommended for individuals with a known heritable syndrome associated with the development of renal cell carcinoma [31, 32]. It has been speculated that more frequent use of cross-section imaging has resulted in these malignancies being diagnosed at earlier stages [33]. In contrast, screening for stomach cancer is better established; however, among older adults testing for Helicobacter pylori infection remains low (7.7\%), even among those who have risk factors associated with this cancer (11.6\%) [34]. While the impacts of screening on the prevalence of the abovementioned cancers is low for all ages [35-37], our study shows that increased diagnoses at early stages impacted both the dynamics of respective cancer prevalences and the trends in relative survival among older patients. Specifically, increasing frequencies of early-stage diagnoses have been shown for cancers of pancreas, kidney, and stomach and 3-year relative survival increased for localized and regional cancers (in 2016 the survival was $20 \%, 75 \%$, and $45 \%$ for pancreatic, kidney, and stomach cancers, respectively; Supplemental Figure 1). On the other hand, for cancers with well recognized screening 
strategies (e.g., for cancers of prostate, breast, and skin melanoma) the contribution of overtime changes in the frequency of early-stage diagnoses to dynamics of prevalence was minimal because such increases over the recent decades were themselves small when compared to the already high rates of early-stage diagnosis for these tumors.

A concerning trend was observed for esophageal cancer which demonstrated a slowing improvement in survival and increasing levels of diagnoses at advanced stages. Both effects substantially contributed to deceleration in cancer prevalence among older U.S. adults. Although recent analysis of SEER data showed that 5-year survival rates for all-age patients with esophageal cancer increased from 1973 to 2010 (for both localized and regional stages), information on survival among older patients remains sparse [38]. It has been speculated that survival of patients with esophageal cancer was slowly improving due to better surgical techniques, adjuvant therapy, and increasing use of upper gastrointestinal endoscopy for early diagnosis [38, 39]. However, it seems that this may not be the case for older patients for whom a deceleration in survival could be associated with changing patterns of histotype-specific incidence with esophageal adenocarcinoma rates surpassing the rates of squamous cell carcinoma [38, 40]. These patterns could contribute to the observed dynamics of early-stage diagnoses. Future studies should address potential causes of changes in survival among older patients with histotype-specific esophageal cancer.

Why are the studies on cancer prevalence trends relatively rare compared to the studies on incidence or mortality? Although many studies focus on cancer mortality trends in the U.S. [4144], studies on cancer prevalence including analyses of dynamics of epidemiologic characteristics that impact prevalence trends are less common [2, 45]. In part, this is due to a lower availability of data on disease prevalence, while mortality data are widely available (e.g., in the Multiple Cause of Death datafiles). Even when the data on cancer prevalence can be obtained (e.g., from SEER-Medicare data), the results requires complex interpretation compared to the results on incidence or mortality [10] because prevalence is based on both disease incidence and its duration, and a high prevalence of disease in a population could reflect high incidence, or better survival, or both (and a low prevalence could indicate low disease incidence, 
or worth survival, or both). Partitioning analysis we used provides: i) high-precision estimates of time trends of cancer prevalence; ii) explanations of these trends based on the trends of cancer incidence, stage-specific relative survival, and ascertainment of cancer at early stages; and iii) interpretation of the obtained results and identification of the groups of cancers with similar patterns. This method reveals the mechanisms of interrelations between cancer incidence, prevalence, and relative survival, thus being an important step in assessing current and future cancer burden, identification of potential problem areas that requires more attention, and evaluation of how these important epidemiological characteristics respond to implemented health interventions over time.

Study limitations. The SEER database used in this study represents approximately $28 \%$ of the U.S. population and underrepresents certain racial/ethnic groups and geographic regions. In this study we investigated cancer sites without further detailing by histotypes; however, cancers of different histotypes (e.g., adenocarcinomas and squamous cell carcinomas, subtypes of ovarian or breast cancers, etc.) are distinct by their risk factors, biology, time trends, populations at risk, treatment, and survival; therefore, their contributions to prevalence trends also differ. Further, our calculations were based on empiric parametric models for partitioning components, and although the models were constructed based on accepted models some statistical uncertainties still remain. In addition, the model for cancer survival in the most recent years can be less precise because of a limited survival time available. SEER data do not provide individual-level variables on cancer risk factors (e.g., smoking and other); therefore, the dynamics of these factors were not integrated into partitioning analysis. Finally, we did not perform gender- and race/ethnicity-specific partitioning analysis in this study to keep it focused on various cancer sites; further studies will include more detailed analyses for these populations subgroups.

Summary. Our analysis shows that the slowing in the increases in the prevalence of many cancers among older U.S. adults over recent decades can be explained by decreasing trends of incidence and improving survival for most cancers, with smaller contributions of the stage at cancer diagnosis. If these dynamics persist, it is likely that cancer burden in older U.S. population of older adults will be lower than projected previously. While decelerating or 
decreasing prevalence of many cancers are due to a beneficial combination of decreasing incidence and increasing survival, there are cancers for which decelerating prevalence is due to the lack of improvement in their stage-specific survival and/or increasing frequency of diagnosis at advanced stages. Overall, if the observed trends persist, it is likely that cancer burden due to its prevalence in older U.S. population will be lower than it was projected in earlier studies. Although the literature on cancer burden and its trends in older populations remains sparse, clinicians are increasingly recognizing the benefits of a geriatric assessment framework in oncology [5, 46], with the complex health needs of older patients and finding efficient ways to meet the medical surveillance needs of older survivors will become increasingly important $[2$, 47]. Although a number of interventions have been developed to help survivors cope with cancer, few have targeted older adults and this remains an area of critical need in survivorship science $[2,47]$. 


\section{Statements and Declarations}

\section{Funding}

This study was supported by the National Institute on Aging (R01-AG066133, RF1-AG046860, R01-AG057801). The sponsors had no role in the design and conduct of this study.

\section{Competing Interests}

The authors have no relevant financial or non-financial interests to disclose.

\section{Author Contributions}

All authors contributed to the study conception and design. Material preparation, data collection and analysis were performed by [Igor Akushevich], [Arseniy Yashkin] and [Mikhail Kovtun]. The first draft of the manuscript was written by [Igor Akushevich] and [Julia Kravchenko] and all authors commented on previous versions of the manuscript. All authors read and approved the final manuscript.

\section{Data Availability}

The datasets generated during and/or analyzed during the current study are available in the National Cancer Institute repository, https://seer.cancer.gov/data/.

\section{Ethics approval}

This study was performed in line with the principles of the Declaration of Helsinki. Approval was granted by the Institutional Review Board of Duke University (FWA00009025), IRB ID Pro00101359.

\section{Consent to participate}

HIPAA Waiver for Informed Consent was approved by the Institutional Review Board of Duke University (FWA00009025).

\section{Consent to publish}

Not Applicable. 


\section{References}

1. De Moor JS, Mariotto AB, Parry C, et al. Cancer survivors in the United States: prevalence across the survivorship trajectory and implications for care. Cancer Epidemiology and Prevention Biomarkers. 2013;22(4):561-570.

2. Bluethmann SM, Mariotto AB, Rowland JH. Anticipating the "silver tsunami": prevalence trajectories and comorbidity burden among older cancer survivors in the United States. Cancer Epidemiology and Prevention Biomarkers. 2016;25(7):1029-1036.

3. Ward MM. Estimating disease prevalence and incidence using administrative data: some assembly required. The Journal of Rheumatology; 2013. p. 1241-1243.

4. Bellizzi KM, Aziz NM, Rowland JH, et al. Double jeopardy? Age, race, and HRQOL in older adults with cancer. Journal of cancer epidemiology. 2012;2012:Article ID 478642.

5. Hurria A, Levit LA, Dale W, et al. Improving the evidence base for treating older adults with cancer: American Society of Clinical Oncology statement. J Clin Oncol. 2015;33(32):38263833.

6. Andes LJ, Li Y, Srinivasan M, Benoit SR, Gregg E, Rolka DB. Diabetes prevalence and incidence among Medicare beneficiaries-United States, 2001-2015. Morbidity and Mortality Weekly Report. 2019;68(43):961-966.

7. Association As. 2019 Alzheimer's disease facts and figures. Alzheimer's \& dementia. 2019;15(3):321-387.

8. O'Neill TW, McCabe PS, McBeth J. Update on the epidemiology, risk factors and disease outcomes of osteoarthritis. Best practice \& research Clinical rheumatology. 2018;32(2):312326.

9. Akushevich I, Yashkin AP, Kravchenko J, et al. Theory of partitioning of disease prevalence and mortality in observational data. Theoretical population biology. 2017;114:117-127.

10. Akushevich I, Yashkin AP, Kravchenko J, et al. Identifying the causes of the changes in the prevalence patterns of diabetes in older US adults: A new trend partitioning approach. $J$ Diabetes Complicat. 2018;32(4):362-367.

11. Akushevich I, Kravchenko J, Yashkin AP, Fang F, Yashin AI. Partitioning of time trends in prevalence and mortality of lung cancer. Statistics in medicine. 2019;38(17):3184-3203.

12. Akushevich I, Yashkin AP, Inman BA, Sloan F. Partitioning of time trends in prevalence and mortality of bladder cancer in the United States. Annals of Epidemiology. 2020;47:25-29.

13. Yabroff KR, Gansler T, Wender RC, Cullen KJ, Brawley OW. Minimizing the burden of cancer in the United States: Goals for a high-performing health care system. CA: a cancer journal for clinicians. 2019;69(3):166-183.

14. Ellis L, Canchola AJ, Spiegel D, Ladabaum U, Haile R, Gomez SL. Trends in cancer survival by health insurance status in California from 1997 to 2014. JAMA oncology. 2018;4(3):317-323. 
15. Kravchenko J, Akushevich I, Abernethy AP, Lyerly HK. Evaluating the Number of Stages in Development of Squamous Cell and Adenocarcinomas across Cancer Sites Using Human Population-Based Cancer Modeling. PloS one. 2012;7(5):e37430.

16. Cho H, Mariotto AB, Mann BS, Klabunde CN, Feuer EJ. Assessing Non-cancer-related health status of US cancer patients: other-cause survival and comorbidity prevalence. American journal of epidemiology. 2013;178(3):339-349.

17. Manton KG, Akushevich I, Kravchenko J. Cancer mortality and morbidity patterns in the US population: an interdisciplinary approach. Springer New York:; 2009.

18. Foster JA, Salinas GD, Mansell D, Williamson JC, Casebeer LL. How does older age influence oncologists' cancer management? The oncologist. 2010;15(6):584-592.

19. Levit LA, Balogh E, Nass SJ, Ganz P. Delivering high-quality cancer care: charting a new course for a system in crisis. National Academies Press Washington, DC; 2013.

20. Unger JM, Coltman Jr CA, Crowley JJ, et al. Impact of the year 2000 Medicare policy change on older patient enrollment to cancer clinical trials. Journal of clinical oncology. 2006;24(1):141-144.

21. Siegel RL, Jacobs EJ, Newton CC, et al. Deaths due to cigarette smoking for 12 smokingrelated cancers in the United States. JAMA internal medicine. 2015;175(9):1574-1576.

22. Thrift AP, El-Serag HB. Burden of gastric cancer. Clinical Gastroenterology and Hepatology. 2020;18(3):534-542.

23. Kruszon-Moran D, Paulose-Ram R, Martin CB, Barker LK, McQuillan G. Prevalence and trends in hepatitis B virus infection in the United States, 2015-2018. 2020;

24. Siegel DA, O’Neil ME, Richards TB, Dowling NF, Weir HK. Prostate cancer incidence and survival, by stage and race/ethnicity-United States, 2001-2017. Morbidity and Mortality Weekly Report. 2020;69(41):1473-1480.

25. Bernard B, Burnett C, Sweeney CJ, Rider JR, Sridhar SS. Impact of age at diagnosis of de novo metastatic prostate cancer on survival. Cancer. 2020;126(5):986-993.

26. Warren JL, Harlan LC, Trimble EL, Stevens J, Grimes M, Cronin KA. Trends in the receipt of guideline care and survival for women with ovarian cancer: a population-based study. Gynecologic oncology. 2017;145(3):486-492.

27. Abdollah F, Gandaglia G, Thuret R, et al. Incidence, survival and mortality rates of stagespecific bladder cancer in United States: a trend analysis. Cancer epidemiology. 2013;37(3):219-225.

28. Zhang L, Sanagapalli S, Stoita A. Challenges in diagnosis of pancreatic cancer. World journal of gastroenterology. 2018;24(19):2047-2060.

29. de Leon AD, Pedrosa I. Imaging and screening of kidney cancer. Radiologic Clinics. 2017;55(6):1235-1250.

30. Canto MI, Harinck F, Hruban RH, et al. International Cancer of the Pancreas Screening (CAPS) Consortium summit on the management of patients with increased risk for familial pancreatic cancer. Gut. 2013;62(3):339-347. 
31. Society AC. American Cancer Society. Cancer Facts \& Figures 2016. 2016. Accessed August 13, 2021. https://www.cancer.org/content/dam/cancer-org/research/cancer-facts-andstatistics/annual-cancer-facts-and-figures/2016/cancer-facts-and-figures-2016.pdf

32. Gray RE, Harris GT. Renal cell carcinoma: diagnosis and management. American family physician. 2019;99(3):179-184.

33. Hollingsworth JM, Miller DC, Daignault S, Hollenbeck BK. Rising incidence of small renal masses: a need to reassess treatment effect. Journal of the National Cancer Institute. 2006;98(18):1331-1334.

34. Florea A, Brown HE, Harris RB, Oren E. Ethnic disparities in gastric cancer presentation and screening practice in the United States: analysis of 1997-2010 surveillance, epidemiology, and end results-medicare data. Cancer Epidemiology and Prevention Biomarkers. 2019;28(4):659-665.

35. Fogel EL, Shahda S, Sandrasegaran K, et al. A multidisciplinary approach to pancreas cancer in 2016: a review. The American journal of gastroenterology. 2017;112(4):537-554.

36. Sung W-W, Ko P-Y, Chen W-J, Wang S-C, Chen S-L. Trends in the kidney cancer mortality-to-incidence ratios according to health care expenditures of 56 countries. Scientific Reports. 2021;11(1):1-8.

37. Kim GH, Liang PS, Bang SJ, Hwang JH. Screening and surveillance for gastric cancer in the United States: is it needed? Gastrointestinal endoscopy. 2016;84(1):18-28.

38. He H, Chen N, Hou Y, et al. Trends in the incidence and survival of patients with esophageal cancer: a SEER database analysis. Thoracic cancer. 2020;11(5):1121-1128.

39. Macías-García F, Domínguez-Muñoz JE. Update on management of Barrett's esophagus. World journal of gastrointestinal pharmacology and therapeutics. 2016;7(2):227-234.

40. Arnal MJD, Arenas ÁF, Arbeloa ÁL. Esophageal cancer: Risk factors, screening and endoscopic treatment in Western and Eastern countries. World journal of gastroenterology: WJG. 2015;21(26):7933-7943.

41. Zahnd WE, James AS, Jenkins WD, et al. Rural-urban differences in cancer incidence and trends in the United States. Cancer Epidemiology and Prevention Biomarkers. 2018. 27(11): p. $1265-1274$.

42. Henley SJ. Invasive cancer incidence, 2004-2013, and deaths, 2006-2015, in nonmetropolitan and metropolitan counties-United States. MMWR Surveillance Summaries. 2017;66(14):1-13.

43. O'Keefe EB, Meltzer JP, Bethea TN. Health disparities and cancer: racial disparities in cancer mortality in the United States, 2000-2010. Frontiers in public health. 2015;3:51.

44. Singh GK, Jemal A. Socioeconomic and racial/ethnic disparities in cancer mortality, incidence, and survival in the United States, 1950-2014: over six decades of changing patterns and widening inequalities. Journal of environmental and public health. 2017;2017:Article ID 2819372. 
45. Phillips SM, Padgett LS, Leisenring WM, et al. Survivors of childhood cancer in the United States: prevalence and burden of morbidity. Cancer Epidemiology and Prevention Biomarkers. 2015;24(4):653-663.

46. Walko CM, McLeod HL. Personalizing medicine in geriatric oncology. Journal of clinical oncology. 2014;32(24):2581-2586.

47. Rowland JH, Bellizzi KM. Cancer survivorship issues: life after treatment and implications for an aging population. Journal of Clinical Oncology. 2014;32(24):2662-2668. 


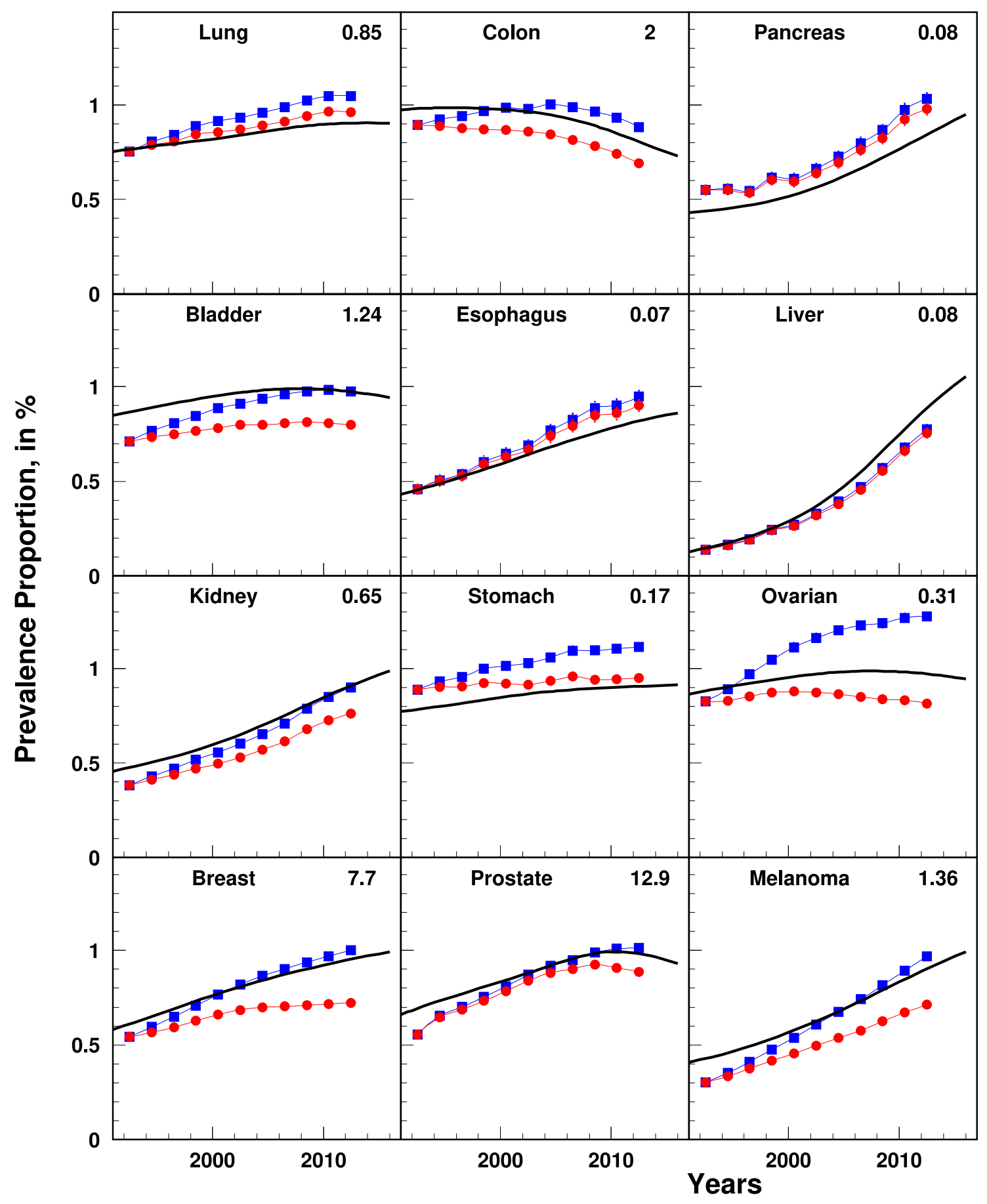

Fig. 1. Estimates of cancer prevalence. Age-adjusted prevalence using our model (solid black line), age-adjusted prevalence using SEER*Stat using an 18-y look-back period (red line with filled circles), and age-adjusted prevalence using SEER*Stat using an up to 23-y look-back period (blue line with filled squares). The value on each plot is a rescaling factor. Actual 
prevalence is obtained by multiplication of this factor and the value taken from Y-axis of the plot.

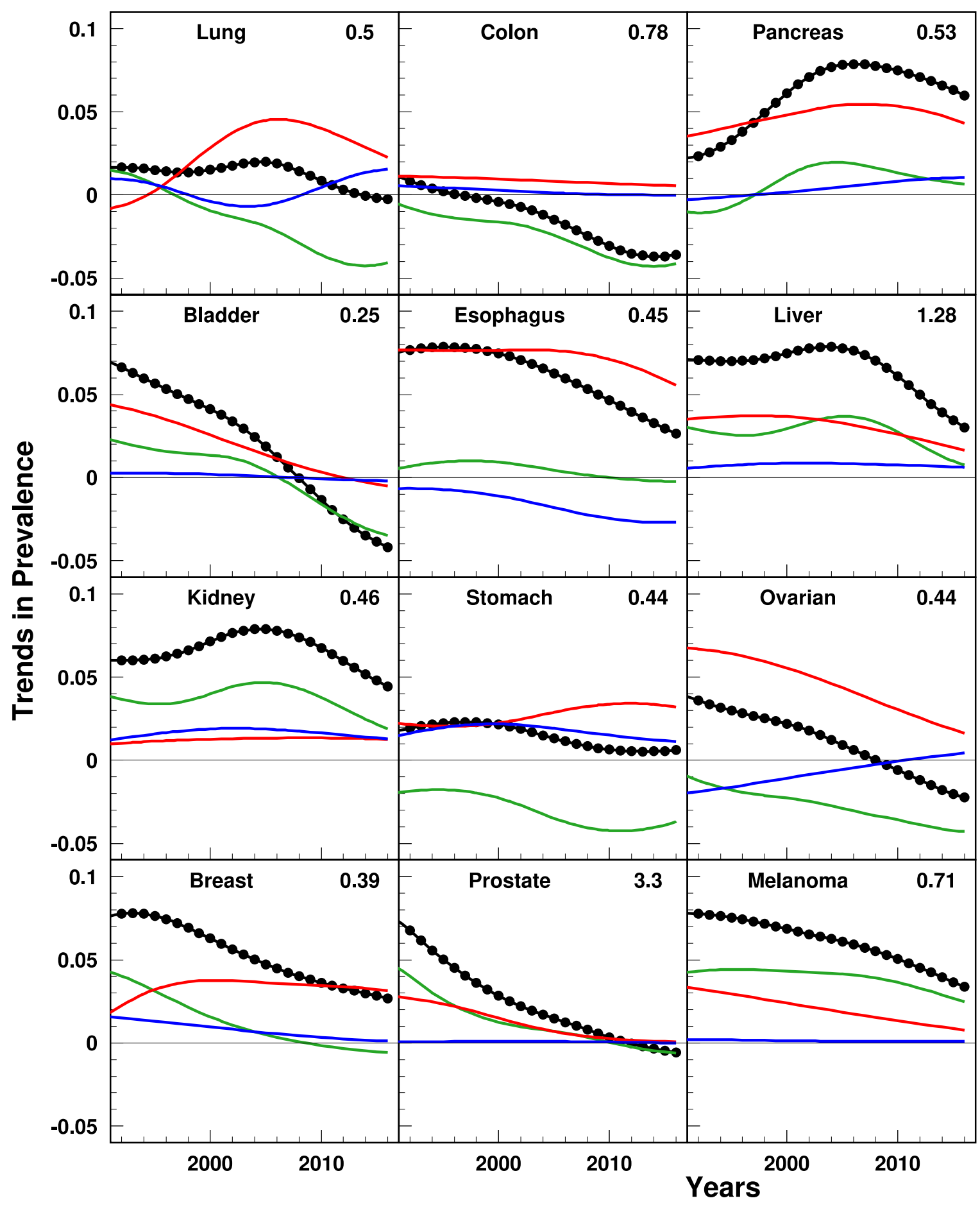

Fig. 2 Partitioning of main cancer age-adjusted prevalence. Color curves represent the contributions of incidence (green), survival (red), and frequency at diagnosis (blue). 


\section{Supplementary Figure 1.}

Empiric patterns for cancers of the study: age-adjusted incidence 65+ (upper left), age-specific incidence averaged over study period (upper right), age-adjusted frequencies of localized, regional, and distant stages (middle left), one- and three-year relative survival for localized (middle right), regional (bottom left) and distant (bottom right) stages. 


\section{Supplementary Files}

This is a list of supplementary files associated with this preprint. Click to download.

- Supplementary.pdf 